\title{
HIGHLIGHTS
}

\section{PEDIATRIC ONCOLOGY}

\section{Fusion-gene status crucial in RMS}

The most common childhood softtissue sarcoma, rhabdomyosarcoma (RMS), is traditionally classified based on morphological characteristics; embroyonal (ERMS) is associated with a better prognosis than alveolar (ARMS). A recent study raises doubts as to the validity of this classification system and challenges the associated risk stratification.

An additional complication is that it appears that guidelines are falling behind clinical practice. Approximately $70 \%$ of ARMS cases are associated with characteristic chromosomal translocations, which form a fusion gene between either $P A X 3$ or $P A X 7$ and FOXO1, the presence of this fusion gene is associated with a poor prognosis. However, the detection of this gene is not currently part of the WHO classification system, although it is used by many clinicians as a supportive diagnostic aid.

The collaborative study was led by Olivier Delattre and Janet Shipley, of Institut Curie and the Institute of Cancer Research, respectively. Delattre described how the research was initiated. "The work ... grew out of a larger project to profile the molecular biology of significant numbers of pediatric tumors ... We felt that RMS, particularly the nature of the two histological subtypes and their relationship to the $P A X$ fusion gene and attention." clinical outcome deserved special

\section{4 ...histological subtype was} not correlated with survival independently of the fusion gene 77

Clinical and molecular characteristics of 210 patients were analyzed; 39 were classified as $P A X$ fusion-gene negative ARMS, 94 as fusion-gene positive ARMS and 77 as ERMS. Delattre described the aim of the study as "to unify expression profiling, comparative genomic hybridization (CGH) data, fusion status and clinical status for a defined set of RMS patients."

The study assessed the fusion-gene status and histological characteristics of all the patients, and whole-genome expression profile and array CGH were performed for 101 and 128 of patients, respectively. Furthermore, the findings were validated on three existing expression datasets by meta-analysis.

The researchers found that "the presence of the PAX/FOXO1 fusion correlated strongly with a poor outcome but that histological subtype was not correlated with survival independently of the fusion gene."

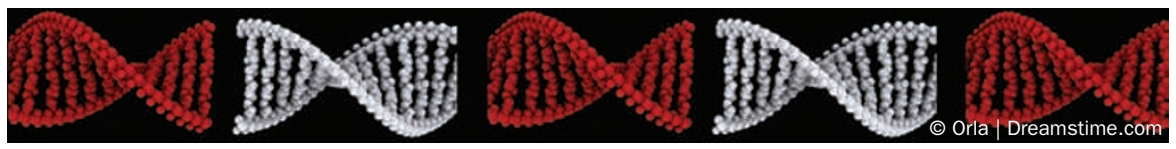

Analysis of the data indicated that there was no significant difference between the event-free survival, overall survival and initial presentation (that is, distribution between favorable and unfavorable sites, age and presence of metastasis) between ARMS negative and ERMS patients. By contrast, ARMS positive patients had a significantly poorer survival, higher frequency of metastasis and unfavorable sites and an older age distribution.

The molecular biology results were described by Delattre: "our expression analysis and CGH analysis showed that the molecular biology of ARMS without $P A X / F O X O 1$ fusions is again indistinguishable from that of ERMS."

Another interesting result in this study was the identification of a characteristic gain of additional copies of chromosome 8 in approximately $70 \%$ of patients with ERMS and fusiongene negative ARMS.

The findings by Delattre and colleagues indicate that the fusion-gene status of patients should be used to guide treatment. Going forward, Delattre hoped and expected that "fusion status will be determined as part of the pathological workup for entry into clinical trials so that these results may be tested across a larger and more closely controlled group." In addition, they plan to assess the role of chromosome 8 in ERMS tumorigenesis.

\section{Rebecca Kirk}

Original article Williamson, D. et al. Fusion gene-negative alveolar rhabdomyosarcoma is clinically and molecularly indistinguishable from embryonal rhabdomyosarcoma.

J. Clin. Oncol. 28, 2151-2158 (2010) 